\title{
Levothyroxine Dose Changes and Hypothyroid Patient Satisfaction-Results of the CONTROL TS Study
}

\author{
McMillan $\mathbf{M}^{1 *}$, Sandulli $\mathbf{W}^{2}$, Engelken $\mathrm{D}^{2}$, Rowe $\mathrm{L}^{3}$ \\ and Bode $B^{3}$ \\ ${ }^{1}$ McMillan Survey Research \& Statistical Consulting, USA \\ ${ }^{2}$ Akrimax Pharmaceuticals, USA \\ ${ }^{3}$ Health Research \& Analytics, USA \\ *Corresponding author: McMillan M, McMillan \\ Survey Research \& Statistical Consulting, Memphis, 8428 \\ Arendal Cove, Memphis, TN, USA
}

Received: June 20, 2017; Accepted: July 24, 2017;

Published: August 08, 2017

\begin{abstract}
Introduction: The effects of levothyroxine dose adjustments on patient satisfaction with drug therapy and physician care have not been described
\end{abstract} previously.

Primary Objective: To measure those effects among: patients who had no changes in levothyroxine dosage within the past year $(n=150)$, and patients who had $\geq 2$ changes in levothyroxine dosage within the past year $(n=150)$.

Methods: Eligible participants completed a 21-question online survey that employed ThyTSQ, a scientifically-validated instrument designed to assess patient satisfaction with: therapy performance and convenience, and the perceived quality of care provided by physicians. Between-group statistical testing was determined using a Chi-square test to compare proportions.

Results: Compared with those reporting no levothyroxine dose changes in the past year, patients reporting $\geq 2$ dose changes were significantly less satisfied with their treatment and treating physician: these patients had lower mean ThyTSQ-Present scores (30.1 vs. 33.2; $P=.002$ ); were less likely to be "very satisfied" with their treatment and dose (22\% vs. $39 \%$; $P=.001)$; and were less likely to feel their hypothyroid symptoms were well controlled $(15 \%$ vs. $34 \% ; P<.001)$. Patients experiencing $\geq 2$ dose changes were also less likely to be "very satisfied" with how their physician dealt with their condition since its diagnosis ( $21 \%$ vs. $34 \% ; P=.015)$, and were more likely to have changed physicians in the past year ( $23 \%$ vs. $14 \%$; $P=.054)$.

Conclusion: Results showed a strong correlation between the frequency of levothyroxine dose changes and reduced patient satisfaction with treatment, treatment convenience, symptom control and physician performance.

Keywords: Dose adjustment; Gastrointestinal; Levothyroxine; Satisfaction; ThyTSQ

\section{Abbreviations}

FT4: FreeT4; IBS: Irritable Bowel Syndrome; GI: Gastrointestinal; GERD: Gastroesophageal Reflux Disease; TSH: Thyroid Stimulating Hormone

\section{Introduction}

Hypothyroidism is a common endocrine disorder resulting from deficiency of thyroid hormone. It is typically a primary process in which the thyroid gland is unable to produce sufficient amounts of thyroxine [1]. In addition to symptoms that are associated with slowing of patients' metabolism, such as weight gain or intolerance to cold, psychological symptoms, including reduced cognitive functioning, are also hallmarks of hypothyroidism [2]. In most cases, hypothyroidism can be treated effectively by oral thyroid hormone supplementation, most commonly levothyroxine.

Hypothyroid patients requiring increased doses of levothyroxine have been well documented in the medical literature and are common in clinical practice [3].

The prevalence and frequency of levothyroxine dose changes were recently confirmed in the CONTROL Surveillance Project conducted among 925 patients treated with levothyroxine monotherapy, the majority of whom (94\%) had been treated for more than 2 years. Among this population, over $47 \%$ reported factors that can affect the performance of levothyroxine. More than 31\% reported having experienced one or more levothyroxine dose changes within the past year; $8 \%$ reported experiencing 2 or more levothyroxine dose changes in the prior 12 months [4].

Although CONTROL Surveillance was not designed to measure quality of life, nearly $11 \%$ of respondents indicated that their thyroid condition "reduced their quality of life" and "rendered them unable to do the things they used to do." Overall, $19.6 \%$ of patients stated that they were not fully satisfied with their hypothyroid treatment. According to Walsh et al. there are anecdotal reports from clinicians of persistent dissatisfaction with levothyroxine therapy among some patients [5]. These results prompted us to examine the association between levothyroxine dose adjustments and patient satisfaction with drug therapy and physician care.

\section{Study objectives}

The primary objective of our study was to measure the effect of
Annals Thyroid Res - Volume 3 Issue 2 - 2017

Submit your Manuscript | www.austinpublishinggroup.com

McMillan et al. (c) All rights are reserved
Citation: McMillan M, Sandulli W, Engelken D, Rowe L and Bode B. Levothyroxine Dose Changes and Hypothyroid Patient Satisfaction-Results of the CONTROL TS Study. Annals Thyroid Res. 2017; 3(2): 109-114. 


Table 1: Demographics of Survey Participants.
\begin{tabular}{|c|c|c|c|}
\hline & Total & $\begin{array}{c}\text { No Dose } \\
\text { Change Group }\end{array}$ & $\begin{array}{c}\text { Dose } \\
\text { Change Group }\end{array}$ \\
\hline Total & 300 & 150 & 150 \\
\hline $\begin{array}{c}\text { Gender } \\
\text { Male } \\
\text { Female }\end{array}$ & $30(10 \%)$ & $\begin{array}{c}17(11 \%) \\
133(89 \%)\end{array}$ & $\begin{array}{c}13(9 \%) \\
137(91 \%)\end{array}$ \\
\hline Age Mean ( \pm SD) & $53.2(14.4)$ & $57.9^{*}(12.88)$ & $48.4(14.34)$ \\
\hline Caucasian/White & $223(74 \%)$ & $105(70 \%)$ & $118(79 \%)$ \\
\hline African American/Black & $55(18 \%)$ & $36(24 \%)$ & $19(13 \%)$ \\
\hline Asian/Asian American & $10(3 \%)$ & $5(3 \%)$ & $5(3 \%)$ \\
\hline Hispanic & $10(3 \%)$ & $3(2 \%)$ & $7(5 \%)$ \\
\hline Native American, Inuit, Aluet & $1(--)$ & $-(-)$ & $1(1 \%)$ \\
\hline $\begin{array}{c}\text { Native Hawaiian/ } \\
\text { Pacific Islander }\end{array}$ & $1(--)$ & $1(1 \%)$ & $--(-)$ \\
\hline
\end{tabular}

${ }^{*} P<.001$, t-test comparing No Dose Change Group with Dose Change Group. Abbreviation: SD: Standard Deviation

levothyroxine dose adjustments among two groups of subjects using a validated treatment satisfaction instrument developed specifically for hypothyroid patients. The first group $(n=150)$ had not experienced any change in levothyroxine dosage or formulation in the prior 12 months (No Dose Change Group). The second group $(\mathrm{n}=150)$ had experienced $\geq 2$ levothyroxine dose or formulation changes in the prior 12 months. Based on decades of clinical experience and studies such as CONTROL Surveillance, we concluded that 2 or more dose changes within the period of a year would be a better indicator of suboptimal therapy than only 1 dose change, which routinely occurs with levothyroxine.

Secondary objectives of the study included a comparison of patient satisfaction between the two groups on several dimensions including overall satisfaction with care, satisfaction with drug therapy and satisfaction with the perceived quality of care provided by treating physicians. The number of study subjects who had changed healthcare providers in the past 12 months was also measured. Patient characteristics were collected for both groups.

\section{Methods}

\section{Sample size calculation}

It was determined that a sample size of 300 would provide $80 \%$ power to detect a difference of $25 \%$ for all satisfaction scores. Statistical testing to compare the two study groups included a Chisquare test to compare proportions.

Selection of patients and exclusion/inclusion criteria: Eligible patients were selected from a proprietary database and asked to complete a 21-question online survey. Patients were excluded from the study if they were $<19$ years old, pregnant or taking levothyroxine for less than one year. For the reasons described under Study Objectives, patients with 1 levothyroxine dose change in the prior year were also excluded. Patients received modest compensation for study participation.

Survey instrument: In order to measure patient satisfaction with hypothyroid treatment, the Underactive Thyroid Treatment Satisfaction Questionnaire (ThyTSQ) was used. This is the first scientifically-validated survey instrument developed to measure treatment satisfaction among hypothyroid patients $[6]^{\star}$. The ThyTSQ survey is divided into two parts, which have been validated as
Table 2: Overall ThyTSQ Scores and Satisfaction Levels.

\begin{tabular}{|c|c|c|c|c|}
\hline & & \multicolumn{2}{|c|}{ Dosage Groups } & \\
\hline & Total & $\begin{array}{c}\text { No Dose Change } \\
\text { Group }\end{array}$ & $\begin{array}{c}\text { Dose } \\
\text { Change Group }\end{array}$ & $\boldsymbol{P}_{\text {-value }}$ \\
\hline Total n & 300 & 150 & 150 & \\
\hline Overall ThyTSQ & 48.3 & 50.9 & 45.8 & $<.001$ \\
\hline Low Satisfaction & 33.9 & 35.8 & 32.6 & .059 \\
\hline High Satisfaction & 55.7 & 56.5 & 54.8 & .079 \\
\hline
\end{tabular}

*t-test comparing No Dose Change Group with Dose Change Group. Table 3: ThyTSQ Scores.

\begin{tabular}{|c|c|c|c|}
\hline \multicolumn{3}{|c|}{ Mean Sum Scores (Scale of 0-6) } & \\
\hline ThyTSQ Mean Scores & $\begin{array}{c}\text { No Dose } \\
\text { Change } \\
\text { Group }(\mathbf{n = 1 5 0 )}\end{array}$ & $\begin{array}{c}\text { Dose Change } \\
\text { Group } \\
(\mathbf{n}=150)\end{array}$ & $\boldsymbol{P}^{- \text {value }^{*}}$ \\
\hline Total ThyTSQ-PAST Mean Score & 17.7 & 15.6 & .002 \\
\hline $\begin{array}{c}\text { Total ThyTSQ-PRESENT Mean } \\
\text { Score }\end{array}$ & 33.2 & 30.1 & .002 \\
\hline
\end{tabular}

*t-test comparing No Dose Change Group with Dose Change Group.

separate instruments: 4 questions measuring treatment and provider satisfaction at the beginning of hypothyroid treatment (ThyTSQPast). An additional 7 questions measure current treatment and provider satisfaction (ThyTSQ-Present). An Overall Satisfaction Score is derived by combining the results of both sections of the survey. The full list of survey questions can be found in the Appendix. For each survey question, the scale is $0-6$, with higher scores representing higher satisfaction. As described above, the scores for all questions are then summed to create the Overall Satisfaction Score.

\section{Data collection}

Responses from 300 survey respondents were stored in an electronic database from which they were abstracted and analyzed. The study database was created and maintained by Health Research \& Analytics (Parsippany, NJ).

\section{Statistical analysis}

Statistically significant differences between the two groups were determined using a Chi-square test to compare proportions. Results were calculated for both sections of the survey (ThyTSQ-Past and ThyTSQ-Present questions) as well as for overall results.

\section{Study ethics/institutional review board information}

The study protocol and methods used in CONTROL TS, including use of the Thy-TSQ survey instrument, were reviewed and approved by the IntegReview IRB in Austin, TX. To ensure that the research was in compliance with the Health Insurance and Accountability Act of 1996 (HIPPA), a waiver of authorization was obtained according to 45 CFR 164.512 (i)(1)(i), which allowed the collection and analysis of protected health data without the authorization of research participants. Survey results for individual patients were blinded to the study sponsor.

\section{Results}

\section{Demographics}

Mean age of patients receiving levothyroxine monotherapy was 53.2 years. Respondents were mostly female: 270 (90\%). Nearly threequarters of respondents were Caucasian (Table 1). Although the demographic characteristics were similar between the two treatment groups, participants in the Dose Change Group were, on average, 9.5 
Table 4: Results measuring satisfaction with initial hypothyroid therapy (ThyTSQ-Past).

\begin{tabular}{|c|c|c|c|}
\hline & $\begin{array}{l}\text { No Dose Change } \\
\text { Group }(n=150)\end{array}$ & $\begin{array}{l}\text { Dose Change } \\
\text { Group }(n=150)\end{array}$ & $P$-value \\
\hline $\begin{array}{l}\% \text { of patients reporting high satisfaction with initial physician communication about the diagnosis of } \\
\text { hypothyroidism }\end{array}$ & $77 \%$ & $65 \%$ & .032 \\
\hline$\%$ of patients reporting high satisfaction with the information from providers about available treatment options & $75 \%$ & $63 \%$ & .019 \\
\hline
\end{tabular}

*t-test comparing No Dose Change Group with Dose Change Group.

Table 5: Importance of patient-reported symptom control ("How well do you feel the treatment is working?").

\begin{tabular}{|c|c|c|c|c|}
\hline & $\begin{array}{c}\text { Total } \\
\text { Respondents }\end{array}$ & $\begin{array}{c}\text { How Well } \\
\text { Treatment } \\
\text { is Working } \\
\text { (Low } \\
\text { Satisfaction) }\end{array}$ & $\begin{array}{c}\text { How Well } \\
\text { Treatment } \\
\text { is Working } \\
\text { (High } \\
\text { Satisfaction) }\end{array}$ & P-value $^{*}$ \\
\hline $\begin{array}{c}\text { Overall Thy } \\
\text { TSQ } \\
\text { Mean Score }\end{array}$ & 48.3 & 33.5 & 52.8 & $<.001$ \\
\hline
\end{tabular}

*t-test of Low Satisfaction Group vs. High Satisfaction Group.

years younger than those in the No Dose Change Group $(P<.001)$. In terms of gender, both study groups were comparable, and reflect the composition of the general hypothyroid population.

Similar to prior studies, subjects in CONTROL TS had a high prevalence of concomitant GI conditions that can adversely affect levothyroxine performance: GERD (34\%); lactose intolerance (11\%); IBS (8\%); GI surgery (6\%); and $H$. pylori infection (2\%).

Food allergies were reported by $9.7 \%$ of participants. Branded formulations were used by $55.7 \%$ of patients; while $44.3 \%$ used generic formulations. Primary care physicians treated $72.3 \%$ of all patients, while specialists treated $23 \%$. PCPs treated $79.3 \%$ of patients in the No Dose Change Group and $65.3 \%$ of those in the Dose Change Group.

\section{Overall results}

The Overall Satisfaction Score was statistically higher among respondents in the No Dose Change Group versus those in the Dose Change Group (50.9 vs. 45.8; $P<.001$, Table 2). Patients in the No Dose Change Group reported higher mean satisfaction scores both on the ThyTSQ-Past questions (17.7 vs. 15.6; $P=.002)$ and the ThyTSQ-Present questions (33.2 vs. 30.1; $P=.002$, Table 3). Overall, $22 \%$ of patients stated that they were not fully satisfied with their hypothyroid treatment.

\section{Results ThyTSQ-Past Questions}

Among questions measuring satisfaction with initial hypothyroid therapy, the following additional observations were made:

- Satisfaction with initial physician communication about the diagnosis of hypothyroidism: Patients in the No Dose Change Group were more likely to report higher levels of satisfaction on this parameter than patients in the Dose Change Group (77\% vs. 65\%; $P$ $=.032$, Table 4).

- Satisfaction with the information from providers about the disease: Patients in the No Dose Change Group were more likely to report higher levels of satisfaction on this parameter than those in the Dose Change Group (69\% vs. 56\%; $P=.018$, Table 4)

- Satisfaction with the information from providers about available treatment options: Patients in the No Dose Change
Group were more likely to report higher levels of satisfaction on this parameter than those in the Dose Change Group ( $75 \%$ vs. $63 \%$; $P=$ .019 , Table 4).

\section{Results: ThyTSQ-Present Questions}

Among questions measuring satisfaction with current hypothyroid treatment, the following additional observations were made:

- Satisfaction with current therapy: A significantly higher percentage of patients in the No Dose Change Group expressed the highest level of satisfaction with their current treatment compared with those in the Dose Change Group (35\% vs. 21\%; $P=.011$, Figure $1)$.

- Efficacy of current treatment: Thirty-one percent of patients in the No Dose Change Group felt that their treatment was working very well, compared to $13 \%$ in the Dose Change Group ( $P$ $<.001)$.

- Ability to control hypothyroid symptoms: Thirty-four

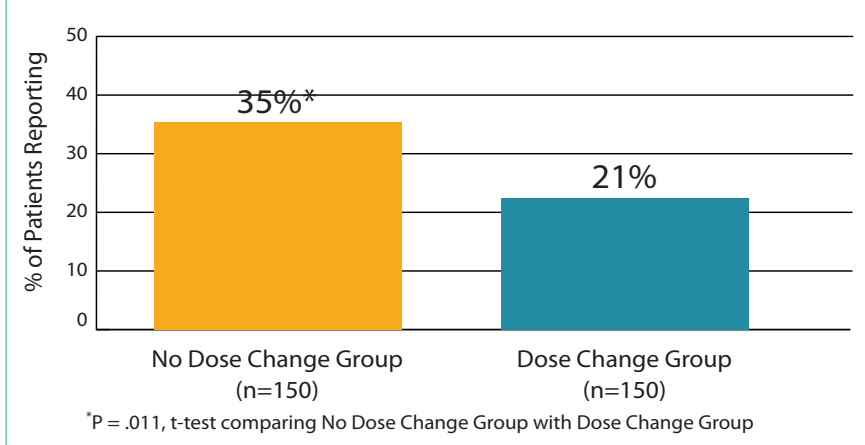

Figure 1: ThyTSQ-Present: Highest Level of Satisfaction with Current Treatment (score=6) ("How satisfied are you with the current treatment for your underactive thyroid?").

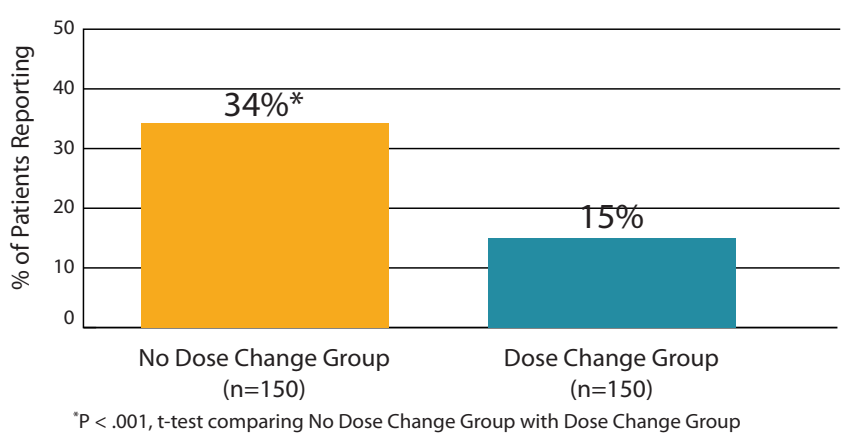

Figure 2: ThyTSQ-Present: Highest Level of Symptom Control (score=6) ("How well do you feel that the treatment is controlling symptoms of underactive thyroid?"). 


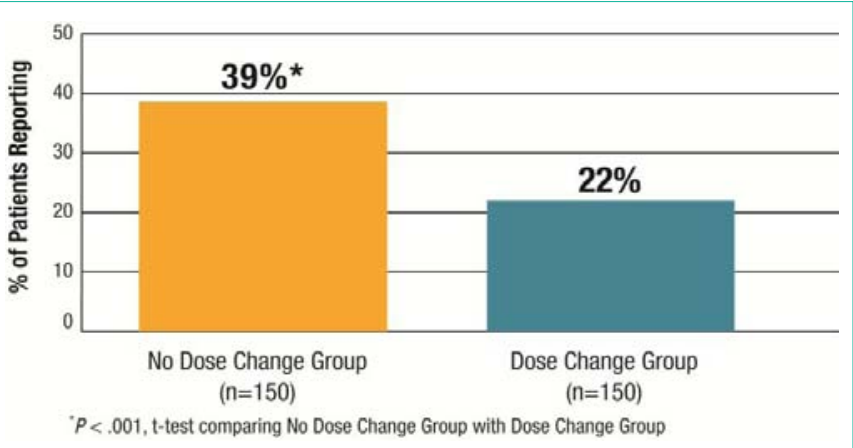

Figure 3: ThyTSQ-Present: Highest Level of Satisfaction with Present Treatment and Dose (score =6) ("How satisfied would you be to continue with your present treatment and dose?").

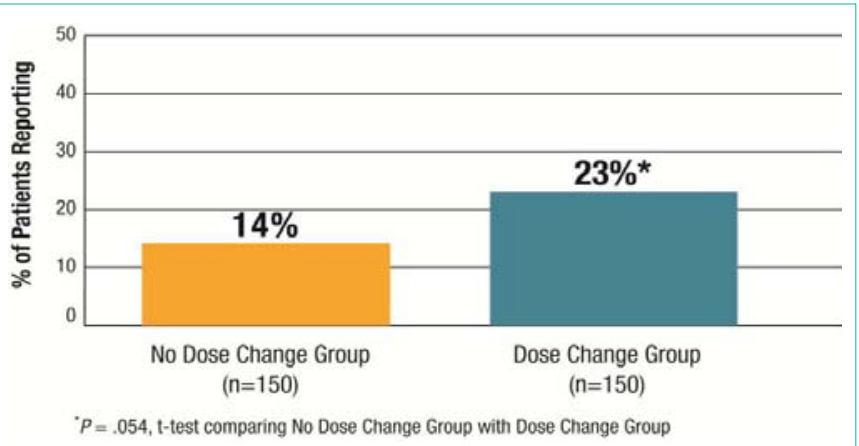

Figure 4: Respondents who Changed Physicians Treating Their Hypothyroidism in Past Year.

percent of patients in the No Dose Change Group felt that their treatment is controlling symptoms very well versus only $15 \%$ in the Dose Change Group $(P<.001$, Figure 2$)$

- Satisfaction with the idea of continuing present therapy: As seen in Figure 3, 39\% of patients in the No Dose Change Group were very satisfied with continuing their present treatment and dose versus $22 \%$ in the Dose Change Group $(P=.001)$.

\section{Changes in treating physician}

The majority of respondents in both treatment groups reported that they had not changed their treating physician in the past year $(82 \% ; 245 / 300)$. However, a substantially higher percentage of patients in the Dose Change Group indicated that they had changed the physician treating their hypothyroidism in the past year versus those in the No Dose Change Group (23\% vs. $14 \%$; $P=.054$, Figure $4)$.

\section{Other Findings}

A secondary objective of CONTROL TS was to examine patient characteristics that may help explain differences in treatment satisfaction among patients taking levothyroxine. Among patients participating in the study, the following were observed:

- $\quad$ Patients with Comorbidities. There was a greater prevalence of comorbid GI conditions among patients in the Dose Change Group versus the No Dose Change Group (52.7\% vs. 46\%; $P=.25$ ).

- $\quad$ Patients with Food Allergies. Eleven percent of patients taking branded formulations of levothyroxine reported having food allergies or celiac disease compared with $8 \%$ of those taking generic levothyroxine. The difference was not statistically significant.

- Physician Specialty and Patient Satisfaction. As noted earlier, primary care physicians were responsible for treating most of the patients enrolled in CONTROL TS (72\%). The percentage of patients treated by endocrinologists in the Dose Change Group was significantly higher than that observed in the No Dose Change Group (31\% vs. $13 \% ; P<.001)$

\section{Discussion}

Levothyroxine has been the "gold standard" for treating hypothyroidism for over 60 years. It is one of the most frequently used medications in the US with over 115 million prescriptions dispensed in 2013 [7]. In spite of this extensive clinical experience, the use of levothyroxine can be challenging. In a survey of 925 patients on levothyroxine monotherapy, McMillan et al. reported that over 31\% of patients had experienced one or more levothyroxine dose changes in the prior 12 months. Among these patients, the majority (60\%) stated that they had experienced one such change; $40 \%$ reported 2 or more changes [4].

Compared with those reporting no levothyroxine dose changes in the past year, patients in our study who reported $\geq 2$ dose changes were significantly less satisfied with their treatment and treating physician: these patients had lower mean ThyTSQ-Present scores (30.1 vs. 33.2; $P=.002)$; were less likely to be "very satisfied" with their treatment and dose; and were less likely to feel their hypothyroid symptoms were well controlled. Patients experiencing $\geq 2$ dose changes were also less likely to be "very satisfied" with how their physician dealt with their condition since its diagnosis, and were more likely to have changed physicians in the past year $(23 \% v s .14 \% ; P=.054)$.

Until now, little was known about the impact of levothyroxine dose changes on patient perceptions of treatment. To our knowledge, CONTROL TS is the first attempt to quantify such perceptions. Its message seems intuitive: medication changes can diminish patient satisfaction with both drug therapy and physician care. However, the issue of patient satisfaction is complex. Patient expectations, physician-patient interaction and other factors inherent to thyroid hormone replacement must be considered. For clinicians wishing to positively influence patient perceptions of care, the following emerge as important conclusions from the results of our study:

\section{Implications for clinicians}

- The Importance of Setting Expectations. Britten et al. have described the self-enforcing outcomes produced by initial expectations of prescribed therapy in terms of both patient satisfaction as well as clinicians' prescribing decisions [8]. Within the context of hypothyroid therapy, this idea carries particular weight as the process of determining optimal levothyroxine dosing can be one of trial and error. This is especially true for patients who may present with factors that have been demonstrated to negatively affect levothyroxine effectiveness (GI disorders, concomitant medication, diet and poor compliance to drug therapy). In CONTROL TS, patients who were less satisfied with their overall therapy were less likely to have been satisfied with the initial information provided about their condition versus those with higher reported levels of satisfaction $(25 \%$ vs. 
$82 \% ; P<.001)$. Compared with all subjects, those reporting low satisfaction with the information provided at first diagnosis also had a significantly lower Overall Satisfaction Score (37.5 vs. 48.3; $P<.001$ ). These patients were also less likely to be satisfied with their initial interactions with healthcare providers (Overall Satisfaction Score: 35.9 vs. $48.3 ; P<.001)$. In our view, the results of CONTROL TS reinforce the need for clinicians to provide clear explanation of what might be expected from thyroid hormone replacement at the outset of treatment. Discussion about the potential need for dose titration as well as factors that may influence levothyroxine absorption such as diet, concomitant GI conditions and the use of other medication may help to avoid patient frustration with therapy and dissatisfaction with treating physicians.

- The Importance of Physician Empathy. The effect of physician empathy on patient satisfaction has been widely reported in the medical literature. Among a study of 550 outpatients at a large university hospital in Korea, Kim et al. reported that "patient-perceived physician empathy significantly influenced patient satisfaction via the mediating factors of information exchange, perceived expertise, inter-personal trust and partnership" [9]. Comstock at al. reported similar findings in a small observational study conducted among internal medicine residents and their patients. The authors reported that "patient satisfaction correlated strongly with ratings for physician courtesy and information-giving". Within the context of hypothyroid treatment, these considerations may be particularly important [10]. In CONTROL TS, patients reporting low levels of satisfaction with "how seriously doctors took them and their hypothyroidism" at the outset of their treatment had lower Overall Satisfaction Scores than those who reported higher satisfaction $(32.2 v s .53 .0 ; P<.001)$.

- The Importance of Symptom Control. In CONTROL TS, patients reporting low levels of satisfaction with "how well their current treatment is working" [to control their hypothyroid symptoms] had significantly lower Overall Satisfaction Scores than those who felt their treatment was working better (33.5 vs. 52.8; $P$ $<$.001) (Table 5). Compared with all subjects, those reporting low satisfaction with how their therapy is currently working to control their hypothyroid symptoms also had significantly lower Overall Satisfaction Scores (33.5 vs. 48.3; $P<.001)$.

These observations reinforce the notion that effective hypothyroid therapy requires an approach that considers both laboratory test findings and patient-reported symptomology. However, the importance of patient feedback must be viewed within the context of the multiple physical and psychological symptoms that can be produced by hypothyroidism. Patients can attribute a wide variety of physical and physiologic changes to their hypothyroidism. However, clinicians who ignore or minimize patient concerns risk incurring poor treatment outcomes. Benvenga et al. have reported that thyroid hormone therapy frequently fails when caregivers do not develop a complete picture of their patient's clinical profile, lifestyle and perceptions of their disease, including its symptoms, via a comprehensive initial screening process and patient dialog [11]. For this reason, caregivers are encouraged to balance laboratory findings with patient perceptions of their own well-being when treating hypothyroidism.

- The Importance of Dose Changes to Therapy Adherence.
While CONTROL TS did not measure the influence of levothyroxine dose changes on therapy adherence, significantly fewer patients in the Dose Change Group were likely to perceive their therapy as being convenient than those in the No Dose Change Group (83\% vs. 95\%; $P=.002)$. This relationship is intuitive as changes to levothyroxine therapy require laboratory tests and follow-up physician visits. Siegal and Greenstein have reported a negative association between medication adherence and the need to take time off from work for treatment among patients being treated for renal disease [12]. In a longitudinal study of 775 patients with Type- 2 diabetes, Balkrishnana et al. described a negative association with long wait times for physician appointments and adherence to prescribed anti-diabetic therapy [13]. Among a study of hypothyroid patients taking levothyroxine therapy for 24 months, Ernst et al. reported significant loss of work productivity associated with frequent changes in levothyroxine therapy [14].

- The Importance of Treatment Satisfaction to Patient Loyalty. While there has been considerable debate among public health experts about patient satisfaction as a measure of healthcare quality, there is strong data to link patient satisfaction with measures that are important to physicians. In the Medical Outcomes Study among 17,000 US adults, Rubin et al. reported that physicians with patient ratings in the lowest 20 percent were almost four times more likely to experience patient turnover than physicians in the top 20\% [16]. In CONTROL TS, Overall Satisfaction Scores were significantly lower in patients who changed physicians in the past year compared with those who did not (43.6 vs. 49.4; $P=.002$ ). Moreover, as noted above, a substantially higher percentage of patients in the Dose Change Group indicated that they had changed the physician treating their hypothyroidism in the past year versus those in the No Dose Change Group (23\% vs. $14 \% ; P=.054)$. Although these results suggest that patients experiencing more than routine levothyroxine dose changes are more likely to change physicians, this correlation does not by itself establish whether changes in treating physicians were caused by dose changes. It is possible, for example, that in some patients, dose changes could have followed the switch to a different physician. However, clinicians should suspect that frequent levothyroxine changes may lead to dissatisfaction and turnover among their patients.

\section{Study Limitations}

Even though adequately powered, the size of the CONTROL TS Study (300 patients) limits its ability to offer a complete picture of the levothyroxine-taking population and their levels of satisfaction with their therapy. The study represents a cohort of patients, mostly female Caucasians, who were treated for hypothyroidism in ambulatory settings. Pregnant patients, patients who have undergone thyroidectomy and pediatric patients were not represented. Our study did not control for gender or measure differences between male and female patients in study parameters. This decision was made because of the study's primary focus on the impact of dose changes and the fact that, in general, a number of patient characteristics, but not gender, affects patient satisfaction. In addition, the vast majority of hypothyroid patients, both in clinical practice and in our study, are female, rendering gender comparisons moot. Given its limited size, our study was not powered to detect differences based on factors such as use of specific thyroid medications or type of treating 
physician (specialist $v s$. non-specialist). Equally important, the study was not designed to determine the reasons why patients may have experienced levothyroxine dose changes in the first place. CONTROL TS should be viewed as neither hypothesis-generating nor conclusive in its findings for these reasons. However, it does provide useful information about how patients perceive their experiences with their hypothyroid treatment and their hypothyroid-treating physicians.

\section{Conclusion}

In CONTROL TS, patients reporting $\geq 2$ levothyroxine dose changes in the past year were significantly less satisfied with their hypothyroid treatment than those reporting no dose changes. Those patients were less likely to feel that their thyroid medication was controlling their hypothyroid symptoms, were less enthusiastic about continuing their current medication regimen and more likely to have changed physicians in the past year.

The results of the CONTROL TS study show a strong correlation between the frequency of levothyroxine dose changes and a reduction in patient satisfaction with treatment, treatment convenience, symptom control and perceived physician performance.

\section{Acknowledgement}

The authors would like to thank Aesculapius Consulting, Inc. (East Brunswick, NJ) for its editorial support.

\section{Foot Note}

${ }^{*}$ Prof. Clare Bradley: 22.7.03 Health Psychology Research Unit, Orchard Building, Royal Holloway, University of London, Egham, Surrey, TW20 OEX. Questionnaires available from www. healthpsychologyresearch.com.

\section{References}

1. Ward LS. The difficult patient: drug interaction and the influence of concomitan diseases on the treatment of hypothyroidism. Arq Bras Endocrinol Metabol. 2010; 54: 435-442.
2. Wiersinga WM. Adult Hypothyroidism. In: Thyroid Disease Manager. 2014

3. Ramadhan A, Tamilia M. Treatment-refractory hypothyroidism. CMAJ. 2012 184: 205-209.

4. McMillan M, Rotenberg KS, Vora K, Sterman AB, Thevathasan L, Ryan MF et al. Comorbidities, concomitant medications, and diet as factors affecting levothyroxine therapy: results of the CONTROL Surveillance project. Drugs RD. 2016; 16: 53-68.

5. Walsh JP. Dissatisfaction with thyroxine therapy - could the patients be right? Curr Opin Pharmacol. 2002; 2: 717-722.

6. McMillan CV, Bradley C, Woodcock A, Razvi S, Weaver JU. Design of new questionnaires to measure quality of life and treatment satisfaction in hypothyroidism. Thyroid. 2004; 14: 916-925.

7. Aitken M, Kleinrock M, Lyle J, Caskey L. Medicine use and shifting costs of healthcare: a review of the use of medicines in the United States in 2013. IMS Institute for Healthcare Informatics. IMS Health. 2014

8. Britten N, Ukoumunne OC, Boulton MG. Patients' attitudes to medicines and expectations for prescriptions. Health Expect. 2002; 5: 256-269.

9. Kim SS, Kaplowitz S, Johnston MV. The effects of physician empathy on patient satisfaction and compliance. Eval Health Prof. 2004; 27: 237-251.

10. Comstock LM, Hooper EM, Goodwin JM, Goodwin JS. Physician behaviors that correlate with patient satisfaction. J Med Educ. 1982; 57: 105-112.

11. Benvenga S. When thyroid hormone replacement is ineffective? Curr Opin Endocrinol Diabetes Obes. 2013; 20: 467-477.

12. Siegal B, Greenstein S. Compliance and noncompliance in kidney transplant patients: cues for transplant coordinators. J Transpl Coord. 1999; 9:104-108.

13. Balkrishnan R, Rajagopalan R, Camacho FT, Huston SA, Murray FT, Anderson RT. Predictors of medication adherence and associated health care costs in an older population with type 2 diabetes mellitus: a longitudinal cohort study. Clin Ther. 2003; 25: 2958-2971.

14. Ernst FR, Barr P, Elmor R, Sandulli W, Thevathasan L, Sterman AB, et al. The Economic Impact of Levothyroxine Dose Adjustments: the CONTROL HE Study. Clin Drug Investig. 2017; 37: 71-83.

15. Rubin HR, Gandek B, Rogers WH, Kosinski M, McHorney CA, Ware JE Jr. Patients' ratings of outpatient visits in different practice settings. Results from the Medical Outcomes Study. JAMA. 1993; 270: 835-840.
Annals Thyroid Res - Volume 3 Issue 2 - 2017

Submit your Manuscript | www.austinpublishing group.com

McMillan et al. (C) All rights are reserved
Citation: McMillan M, Sandulli W, Engelken D, Rowe L and Bode B. Levothyroxine Dose Changes and Hypothyroid Patient Satisfaction-Results of the CONTROL TS Study. Annals Thyroid Res. 2017; 3(2): 109-114. 